\title{
Vaccination in Psoriasis: A Prophylactic Potential or Aa Therapeutic Adjuvant
}

\section{Mohamed L Elsaie*}

Department of Dermatology and Cutaneous Surgery, Miller School of Medicine, University of Miami, Miami, Florida 33140, USA

Patients with immune-mediated inflammatory diseases (IMID, for example RA, IBD or psoriasis, are at expanded danger of tainting, incompletely on account of the malady itself, yet generally as a result of medicine with immunomodulatory or immunosuppressive medications. Notwithstanding their raised hazard for antibody preventable malady, immunization scope in Imid patients is shockingly low. The term insusceptible interceded incendiary malady (IMID) spreads an assembly of evidently pointless maladies influencing different organs and frameworks, for example Ra, Ibd and psoriasis. Then again, these scatters impart some normal hereditary inclinations and incendiary pathways, portrayed by cytokine dysregulation. Subsequently, comparative mitigating medication techniques, incorporating organization of immunosuppressive or immunomodulatory executors (from this point forward named immunotherapy), are utilized to treat these clutters.

Inoculation is a demonstrated and overall secured system for aversion of irresistible infections in the overall public and in patients with Imid, who have an expanded danger of inconveniences for some antibody preventable contaminations, because of both the way of the sickness and its immunomodulatory medicine.

Imid patients, specifically those under immunotherapy, are at an expanded hazard for inconveniences of some immunization preventable contaminations. Thus, for this patient populace the profits of executing a suitable inoculation convention in every day clinical practice are conceivably even more stupendous than for the overall public. The point when inoculation scope in the populace is high, crowd resistance allows a certain degree of assurance to non-immunized people by lessening the pervasiveness of the infection.

Conceivable descriptions for under-immunization of Imid patients are unawareness of the expanded contamination hazard, and worries about security and adequacy of inoculation in this patient bunch. Components to think about when assessing the wellbeing of an antibody in Imid patients are the speculative hazard for a flare of the Imid after immunization and, for live antibodies, the danger of antibody impelled contaminations. The hesitance of clinicians to immunize Imid patients may be because of alarm of antibody actuated illness flares, and to the concern if the more level resistant reaction watched in Imid patients treated with immunomodulatory drugs still gives sufficient security against the ailment [1].

The inquiry is to if such immunizations might serve as a prophylactic or further turn into an adjuvant help in the fight against IMID.

An as of late distributed study by El Darouti et al., assessed the adequacy and wellbeing of live constricted varicella immunization as an adjuvant immunotherapeutic modality in medicine of extreme safe psoriasis. The randomized, placebo regulated, twofold blinded study incorporated 35 patients with intense safe psoriasis. Patients were haphazardly separated into 2 gatherings; aggregate $A$ (18 patients) and bunch B (17 patients) who all accepted cyclosporine at a day by day measurements of $2.5 \mathrm{mg} / \mathrm{kg} /$ day. Likewise, bunch A gained 4 dosages of live constricted varicella immunization once/3 weeks, at the same time gather B accepted 4 measurements of subcutaneous saline in the same design as gathering A. Clinical evaluation was dependent upon the psoriasis territory and intensity file score computation at every visit. Last persistent's reaction (week 12) was appraised as per the medical practitioner worldwide evaluation. Results: Group A demonstrated fundamentally higher \% of change in their Pasi score (mean, 95\% Ci $81.83,69.05-94.60)$ than aggregation B (mean, 95\% Ci 50.12, 34.5865.66) ( $\mathrm{P}=0.002)$. Bunch An accomplished a more huge change in their score assessments than aggregation B. $83.3 \%(15 / 18)$ of assembly An accomplished a weighty change (_50\% change) versus $47.1 \%(8 / 17)$ of aggregation $\mathrm{B}(\mathrm{P}=0.024)$. The examination group encountered negligible unfavorable occasions to their patients and no medication end was required [2].

The creators clarified that the varicella-zoster infection conceivably fortified the humoral reaction that is intervened by T-partner (Th)2 cells, and down-managing the Th- 1 reaction. An alternate prescribed reaction was the down regulation of the Th17 intervened pathogenic impacts through the alpha interferon discharge by the varicella antigen.

The group has executed a guaranteeing positive study be that as it may; further enduring assessment and appraisal of the immunocompetence of such patients is vital remembering thatcontingent upon the level of immunosuppression-the reaction to the antibody and possibly the time of insurance and malady free interims could be restricted in such patients.

\section{References}

1. Rahier JF, Moutschen M, Van Gompel A, Van Ranst M, Louis E, et al. (2010) Vaccinations in patients with immune-mediated inflammatory diseases. Rheumatology (Oxford) 49: 1815-1827.

2. El-Darouti MA, Hegazy RA, Abdel Hay RM, Abdel Halim DM (2012) Live attenuated varicella vaccine: a new effective adjuvant weapon in the battlefield against severe resistant psoriasis, a pilot randomized controlled trial. J Am Acad Dermatol 66: 511-513.

*Corresponding author: Mohamed L Elsaie, Department of Dermatology and Cutaneous Surgery, Miller School of Medicine, University of Miami, Miami, Florida 33140, USA, Tel: 305-243-6734; E-mail: egydoc77@yahoo.com

Received October 28, 2013; Accepted November 02, 2013; Published November 06, 2013

Citation: Elsaie ML (2013) Vaccination in Psoriasis: A Prophylactic Potential or Aa Therapeutic Adjuvant. J Vaccines Vaccin 4: e123. doi: 10.4172/2157$7560.1000 \mathrm{e} 123$

Copyright: $(2013$ Elsaie ML. This is an open-access article distributed under the terms of the Creative Commons Attribution License, which permits unrestricted use, distribution, and reproduction in any medium, provided the original author and source are credited. 